\title{
Difference in glenoid retroversion between two-dimensional axial computed tomography and three-dimensional reconstructed images
}

\author{
Hyungsuk Kim, Chang Hyun Yoo, Soo Bin Park, Hyun Seok Song \\ Department of Orthopedic Surgery, Eunpyeong St. Mary's Hospital, College of Medicine, The Catholic University of Korea, Seoul, Korea
}

\begin{abstract}
Background: The glenoid version of the shoulder joint correlates with the stability of the glenohumeral joint and the clinical results of total shoulder arthroplasty. We sought to analyze and compare the glenoid version measured by traditional axial two-dimensional (2D) computed tomography (CT) and three-dimensional (3D) reconstructed images at different levels.

Methods: A total of 30 cases, including 15 male and 15 female patients, who underwent 3D shoulder CT imaging was randomly selected and matched by sex consecutively at one hospital. The angular difference between the scapular body axis and 2D CT slice axis was measured. The glenoid version was assessed at three levels (midpoint, upper one-third, and center of the lower circle of the glenoid) using Friedman's method in the axial plane with 2D CT images and at the same level of three different transverse planes using a 3D reconstructed image.

Results: The mean difference between the scapular body axis on the $3 \mathrm{D}$ reconstructed image and the $2 \mathrm{D}$ CT slice axis was $38.4^{\circ}$. At the level of the midpoint of the glenoid, the measurements were $1.7^{\circ} \pm 4.9^{\circ}$ on the $2 \mathrm{D}$ CT images and $-1.8^{\circ} \pm 4.1^{\circ}$ in the $3 \mathrm{D}$ reconstructed image. At the level of the center of the lower circle, the measurements were $2.7^{\circ} \pm 5.2^{\circ}$ on the $2 \mathrm{D}$ CT images and $-0.5^{\circ} \pm 4.8^{\circ}$ in the $3 \mathrm{D}$ reconstructed image. A statistically significant difference was found between the 2D CT and 3D reconstructed images at all three levels.

Conclusions: The glenoid version is measured differently between axial 2D CT and 3D reconstructed images at three levels. Use of 3D reconstructed imaging can provide a more accurate glenoid version profile relative to 2D CT. The glenoid version is measured differently at different levels.
\end{abstract}

Keywords: Shoulder; Scapula; Bone retroversion; Multidetector computed tomography; Three dimensional

\section{INTRODUCTION}

The glenoid version of the shoulder joint is correlated with the stability of the glenohumeral joint and clinical results of total shoulder arthroplasty [1-3]. The glenoid version is of interest in shoulder joint pathologies. Posterior instability of the shoulder joint is reported to be associated with increased retroversion of the glenoid $[4,5]$. Bone loss of the glenoid affects the glenoid version, resulting in changes in contact pressure in the glenohumeral joint [6-8]. Also, it is important to evaluate the glenoid inclination before conducting shoulder arthroplasty, since an altered glenoid inclination due to the glenoid component may facilitate

Received: March 17, $2020 \quad$ Revised: May 6, 2020 Accepted: May 7, 2020

Correspondence to: Hyun Seok Song

Department of Orthopedic Surgery, Eunpyeong St. Mary's Hospital, College of Medicine, The Catholic University of Korea, 1021 Tongil-ro, Eunpyeonggu, Seoul 03312, Korea

Tel: +82-2-2030-4628, Fax: +82-2-2030-4629, E-mail: hssongmd@hanmail.net, ORCID: https://orcid.org/0000-0002-7844-2293

\section{Financial support: None.}

Conflict of interest: None.

Copyright@ 2020 Korean Shoulder and Elbow Society. All Rights Reserved.

This is an Open Access article distributed under the terms of the Creative Commons Attribution Non-Commercial License (http://creativecommons.org/licenses/by-nc/4.0/) which permits unrestricted non-commercial use, distribution, and reproduction in any medium, provided the original work is properly cited. 
biomechanical changes in the glenohumeral joint, resulting in different wear patterns of the glenoid component [8-11].

The glenoid version is reported from $2^{\circ}$ anteversion to $9^{\circ}$ retroversion and varies among individuals and races [7]. Under the aid of a scapula-holding device, the glenoid version was measured with a goniometer in several cadaveric studies [7,12,13]. In image studies, the measurement method described by Friedman et al. [6] is mainly used and adopts a line positioned between the medial border of the scapula to the center of the glenoid as a reference line for the scapular axis. Although various methods of measuring the glenoid version have been discussed, methods involving computed tomography (CT) scans, mostly axial two-dimensional (2D) CT images, are preferred and known to be more accurate than those incorporating measurements of plain radiograph images [14]. Recently, reports suggesting new methods of measuring the glenoid version with magnetic resonance imaging (MRI) are comparable to those using CT scans $[15,16]$.

However, the measurement process may be affected by patient posture in a variety of ways, such as if the scapular position is not aligned with the axis of the CT scanner. There is a report suggesting that scapular rotation in the coronal and sagittal planes will affect the variation of the measured glenoid version, highlighting in particular that $0.42^{\circ}$ of anteversion was gained with $1^{\circ}$ of scapular abduction in the coronal plane [17]. Discrepancies between the actual glenoid version and the scans can be attributed to changes in scapula posture.

There are image software programs that offer the capacity for three-dimensional (3D) reconstruction based on 2D CT images. Using these programs, it is possible to measure and analyze the reconstructed image by freely rotating the image in 3D. Moreover, it is possible to print models using $3 \mathrm{D}$ printing technology [18]. In a $3 \mathrm{D}$ reconstructed image, the transverse plane of the glenoid can be decided at various levels. The transverse plane obtained on $3 \mathrm{D}$ reconstructed scans is not altered by the position of the CT scanner or scapula or by the patient's posture.

Our hypothesis was that differences exist between the glenoid version measured with axial $2 \mathrm{D} \mathrm{CT}$ and $3 \mathrm{D}$ reconstructed imaging, respectively. This thought arose from the discrepancy between the axis of the CT scanner and the scapula position, which can be changed by patient posture. Therefore, the purpose of this study was to analyze and compare the glenoid version measured with traditional axial 2D CT and with 3D reconstructed imaging, respectively.

\section{METHODS}

This study was approved by the Institutional Review Board of
Eunpyeong St. Mary’s Hospital (IRB No. PIRB-20200305-005).

\section{Patient Enrollment}

A total of 30 cases, including 15 male and 15 female patients, who underwent shoulder 3D CT was consecutively selected and matched by sex at one hospital. Patients with glenoid fracture, previous shoulder operation, deformity due to severe glenoid wear or erosion, or poor CT scan quality were excluded. The mean age of male patients was 52.5 years (range, $28-72$ years) and that of female patients was 64.0 years (range, $22-84$ years).

\section{Three-Dimensional Reconstruction Protocol}

Shoulder 3D CT (Optima CT 660; GE Healthcare, Chicago, IL, USA) scans were collected with the patient in the supine position. A free open-source software program, ITK snap (version 3.4.0; United States National Institute of Biomedical Imaging and Bioengineering, Bethesda, MD, USA) [19], was used for reconstructing 2D CT images into 3D images (Fig. 1). Meshmixer (version 3.0; Autodesk Inc., San Rafael, CA, USA), a free opensource software program, was used to rotate and edit the $3 \mathrm{D}$ reconstructed images (Fig. 2).

A scapular coronal plane as a reference plane, based on the method introduced by Kwon et al. [13], was achieved by connecting three reference points (i.e., inferior tip of the scapular body, medial pole of the scapula, and center of the glenoid surface). The area orthogonal to this plane was defined as the transverse scapular plane (Fig. 3).

\section{Measuring the Angle between the 2D Axial CT Scan and Scapular Coronal Plane}

The glenoid version can be measured ideally when the 2D CT slice axis is orthogonal to the scapular body axis. The 2D CT slice axis was established by use of the scout image function in a PACS (picture archiving and communication) system (Marosis M-view version 5.4; Marotech, Seoul, Korea). We checked for an angular difference between the scapular body axis and the actual 2D CT slice axis (Fig. 4).

\section{Measuring the Glenoid Version with 2D Axial CT}

The glenoid version was measured at three levels, including the upper one-third of the glenoid, midpoint of the glenoid, and center of the lower circle of the glenoid. A center point bisecting the anatomical glenoid axis (i.e., at the midpoint of the glenoid) was chosen because it represents the base plate position in anatomical total shoulder arthroplasty. The center of the lower circle of the glenoid was chosen because it is the center peg hole of the base plate for reverse total shoulder arthroplasty. On axial 2D CT scans 


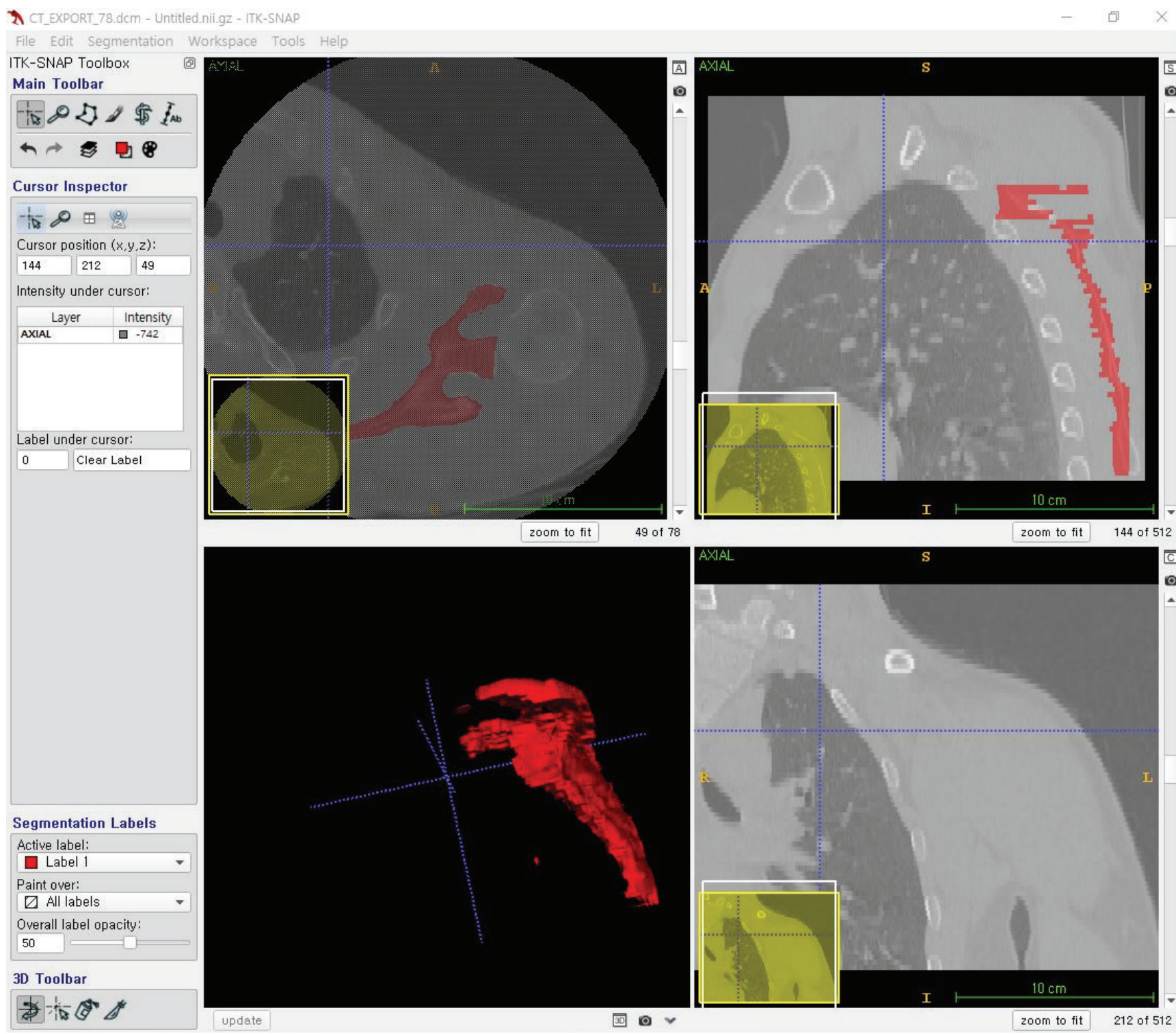

Fig. 1. ITK SNAP (version 3.4.0) was used for reconfiguring computed tomography axial images into the three-dimensional structure image.

of these three levels, the glenoid version was measured (Fig. 5). The measuring method developed by Friedman et al. [6] was adopted at each level [20].

\section{Measuring the Glenoid Version Using 3D Reconstructed Imaging}

Here, a 3D reconstructed image was sliced along the transverse scapular plane orthogonal to the scapular coronal plane. The axial 3D slice was taken at the same three levels as those chosen for assessment on the 2D CT scans (Fig. 6).

\section{Statistical Analysis}

Measured glenoid versions were compared between the 2D CT scans and the $3 \mathrm{D}$ reconstructed image at each of the three levels.
The IBM SPSS ver. 24.0 (IBM Corp., Armonk, NY, USA) was used for all statistical analyses. The Kolmogorov-Smirnov test was used for normal distribution assessments of all measurements. A paired t-test and the signed rank-test were used to compare the glenoid version between the data from 2D CT scans and 3D reconstructed images, respectively. An analysis of variance and the Kruskal-Wallis test were chosen to compare the glenoid version between the 2D CT images and $3 \mathrm{D}$ reconstructed image at each level. Significance levels for all analyses were set at $\mathrm{P}<0.05$.

\section{RESULTS}

The mean angle between the 2D CT slice axis and the scapular body axis on the $3 \mathrm{D}$ reconstructed image was $38.4^{\circ}$. The mean 


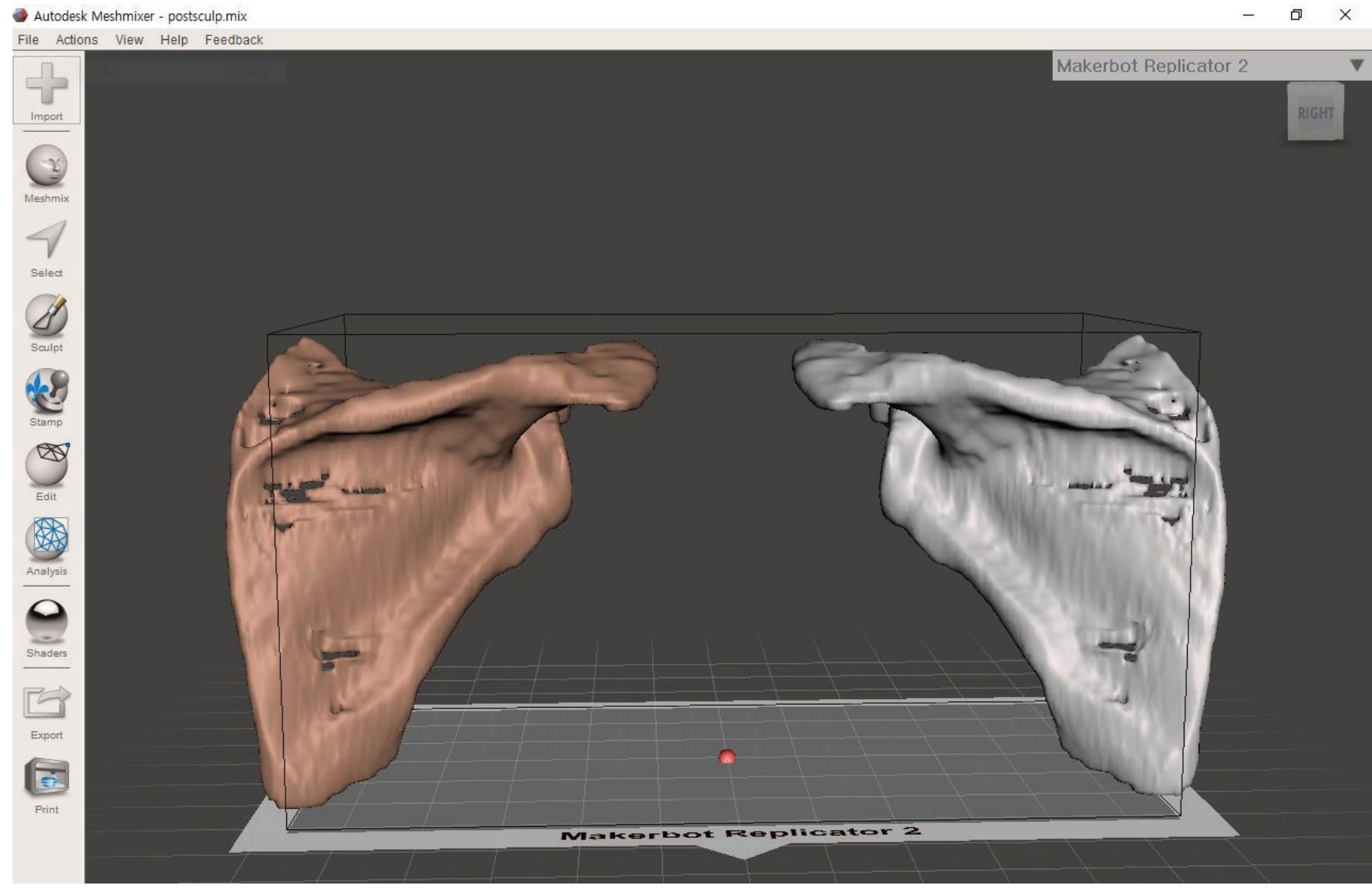

Fig. 2. Meshmixer (version 3.0) was used to edit and rotate the three-dimensional reconstructed image.
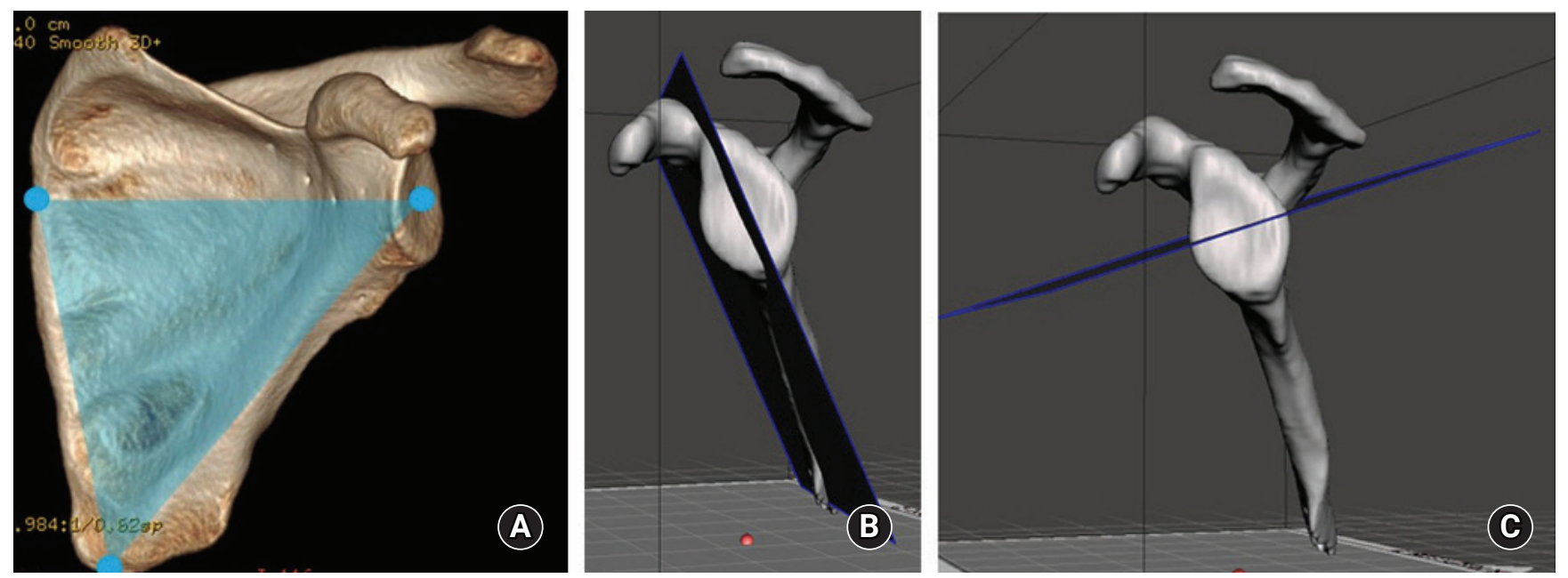

Fig. 3. Scapular plane. (A) The scapular coronal plate, as the reference plane, was defined by connecting the inferior tip of the scapular body, medial pole of the scapula, and the midpoint of the glenoid surface. (B) The scapular coronal plane was demonstrated on the three-dimensional constructed image with the Meshmixer software. (C) The transverse scapular plane was marked orthogonally to the scapular coronal plane.

glenoid version measured at the level of the upper one-third of the glenoid was $-3.6^{\circ} \pm 4.5^{\circ}$ on the $2 \mathrm{D}$ CT images and $-4.5^{\circ} \pm 5.7^{\circ}$ on the $3 \mathrm{D}$ reconstructed image. At the level of the glenoid center, the glenoid version was measured as $1.7^{\circ} \pm 4.9^{\circ}$ on the $2 \mathrm{D}$ CT images and $-1.8^{\circ} \pm 4.1^{\circ}$ on the $3 \mathrm{D}$ reconstructed image. Finally, the mean glenoid version measured at the level of the center of the lower circle of the glenoid was $2.7^{\circ} \pm 5.2^{\circ}$ on the $2 \mathrm{D}$ CT images and $-0.5 \pm 4.8^{\circ}$ on the $3 \mathrm{D}$ reconstructed image. A statisti- 


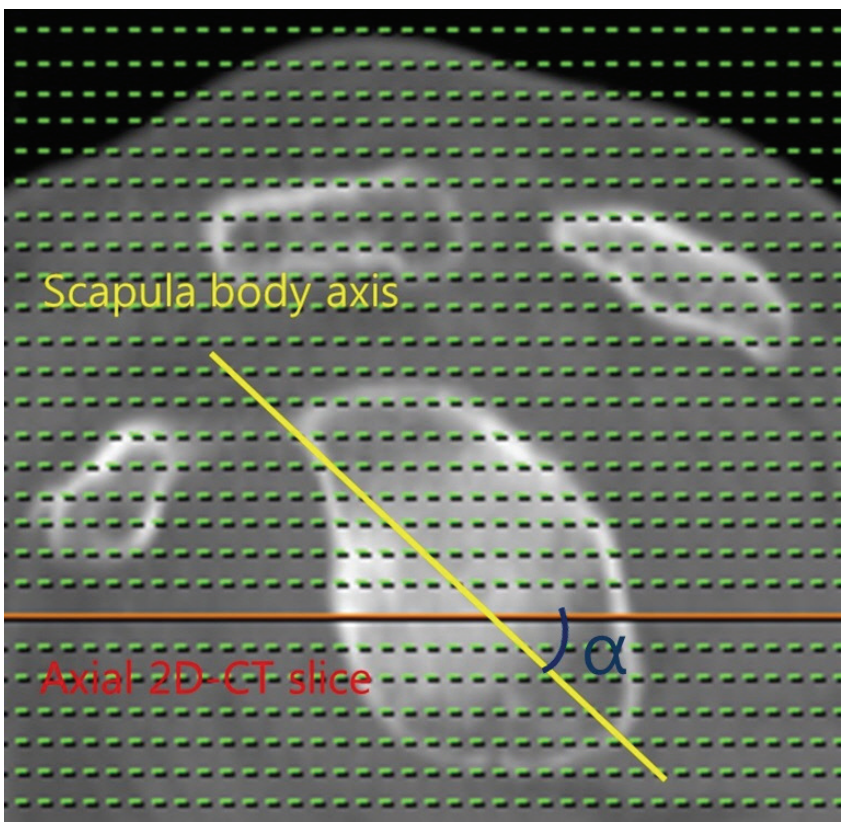

Fig. 4. The angle between the axis of a standard axial two-dimensional computed tomography (2D CT) scan (orange line) and the scapula body axis (yellow line) was measured. cally significant difference was found between the glenoid version measured on the $2 \mathrm{D}$ CT images and $3 \mathrm{D}$ reconstructed image at all three levels $(\mathrm{P}=0.003, \mathrm{P}=0.000$, and $\mathrm{P}=0.000$, respectively) (Table 1).

Considering results of the same modality, a statistical difference was found among the three levels between 2D CT scans $(\mathrm{P}=0.000)$ and $3 \mathrm{D}$ reconstructed images $(\mathrm{P}=0.007)$. During a multiple comparison test, a statistical difference was found between the upper one-third of the glenoid and the midpoint of the glenoid $(\mathrm{P}=0.000)$ and between the upper one-third and the center of the lower circle of the glenoid $(\mathrm{P}=0.000)$ on the $2 \mathrm{D} C \mathrm{CT}$ images. During a multiple comparison test of the $3 \mathrm{D}$ reconstructed image, a statistical difference was found between the upper one-third of the glenoid and the center of the lower circle of the glenoid $(\mathrm{P}=0.006)$. All angles were measured by two orthopedic surgeons (HK and HSS) independently. The interclass correlation coefficient was greater than 0.9 .
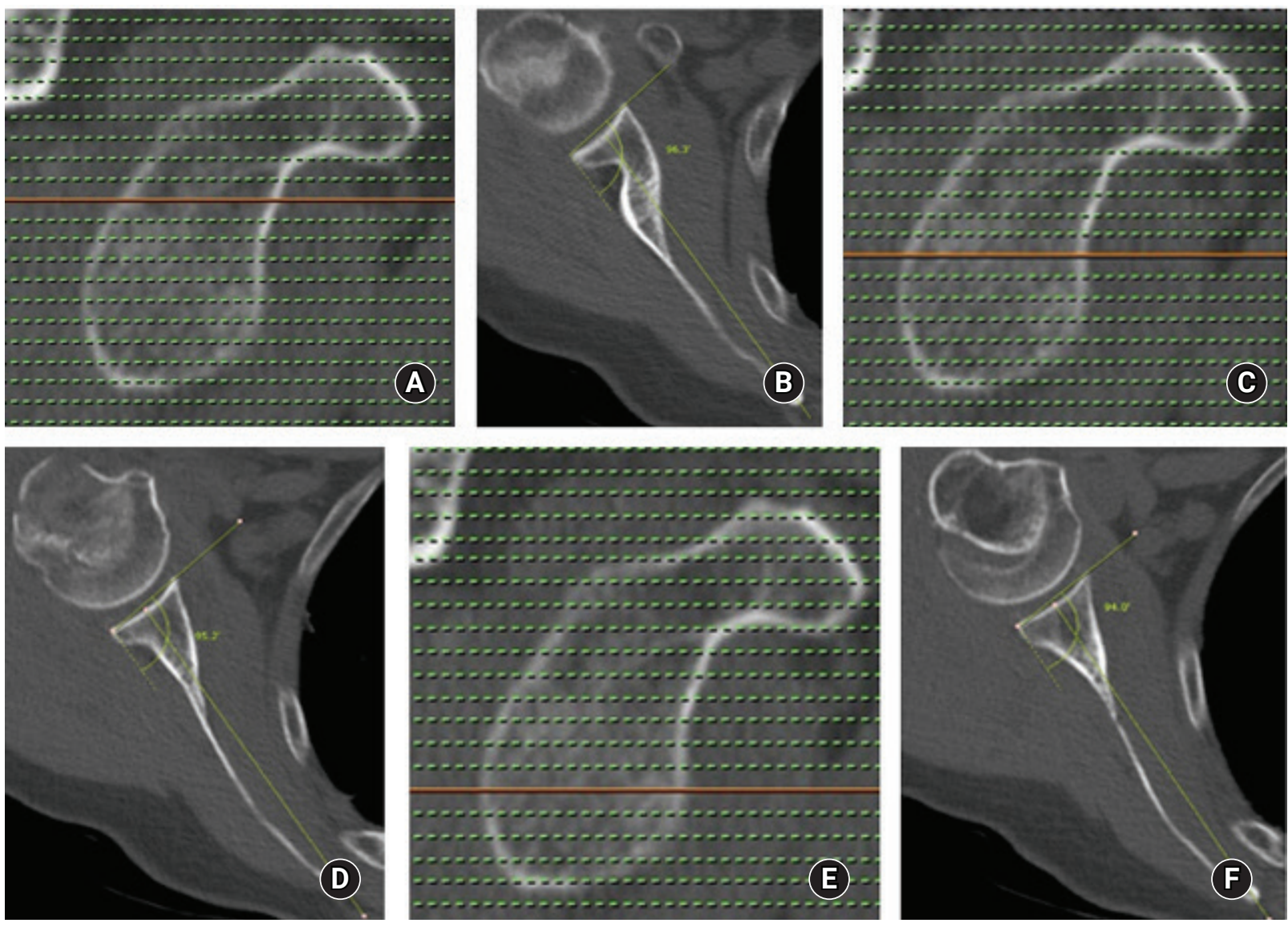

Fig. 5. The glenoid version as measured on an axial two-dimensional computed tomography scan. (A) The level of measurement at the upper one-third of the glenoid. (B) The glenoid version at the upper one-third of the glenoid. (C) The level of measurement at the center of the glenoid. (D) The glenoid version at the center of the glenoid. (E) The level at the center of the lower plane. (F) The glenoid version at the center of the lower circle of the glenoid. 

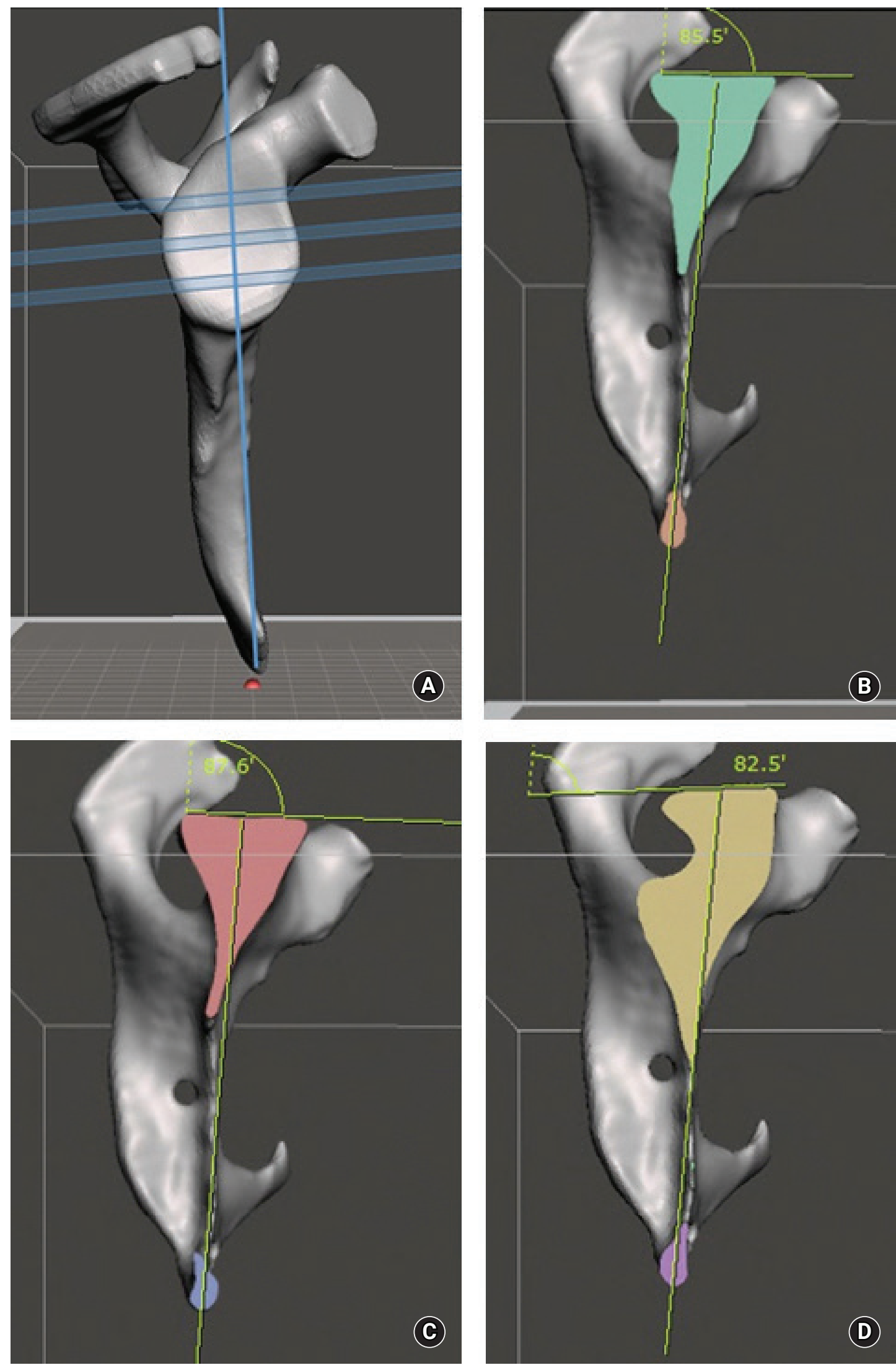

Fig. 6. The glenoid version as measured on the three-dimensional (3D) reconstructed image. (A) The 3D reconstructed image showing three transverse planes. (B) The glenoid version as assessed at the upper one-third transverse plane. (C) The glenoid version as assessed at the center of the glenoid. (D) The glenoid version as assessed at the center of the lower circle of the glenoid. 
Table 1. Mean glenoid version on the 2D CT images and 3D reconstructed image (minus value represents retroversion)

\begin{tabular}{lccc}
\hline Level of the glenoid & 2D CT & 3D reconstruction & P-value* \\
\hline Upper one-third $\left(^{\circ}\right)$ & $-3.6 \pm 4.5$ & $-4.5 \pm 5.7$ & 0.003 \\
Center $\left(^{\circ}\right)$ & $1.7 \pm 4.9$ & $-1.8 \pm 4.1$ & 0 \\
Center of the lower circle $\left(^{\circ}\right)$ & $2.7 \pm 5.2$ & $-0.5 \pm 4.8$ & 0 \\
P-value $^{\dagger}$ & 0 & 0.007 & \\
\hline
\end{tabular}

Values are presented as mean \pm standard deviation.

2D: two-dimensional, CT: computed tomography, 3D: three-dimensional.

${ }^{\star}$ Paired t-test. ${ }^{\dagger}$ Analysis of variance.

\section{DISCUSSION}

In this study, there was a significant difference in glenoid version between measurements collected from routine axial 2D CT scans and a $3 \mathrm{D}$ reconstructed image at three levels of the glenoid. Thus, the glenoid version could be different depending on the level of measurement and whether $2 \mathrm{D}$ axial CT scans or a $3 \mathrm{D}$ reconstructed image is assessed. Restoring the glenoid version anatomically is important when performing shoulder arthroplasty since it affects the clinical results [21,22]. It is known that the possibility of loosening increases when the glenoid component is inserted in a retroversion position of more than $10^{\circ}[8,23]$. To restore the glenoid version accurately, it is important to measure and analyze the glenoid version preoperatively.

In most of the available reports in the literature, the glenoid version was measured on axial 2D CT scans. In the study by Nyffeler et al. [14], which compared the glenoid version as measured in the axillary view of a plain radiograph and an axial 2D CT scan image, the glenoid version was measured more accurately on the CT image. However, there could be a discrepancy depending on the position of the CT scanner and the patient's scapula when the image was taken [24]. In the present study, the mean angle between the 2D CT slice axis and the scapular body axis on the $3 \mathrm{D}$ reconstructed image was $38.4^{\circ}$.

To overcome this discrepancy, Kwon et al. [13] measured the glenoid version on 3D reconstructed images of cadaveric scapulae and suggested that measuring the glenoid version on a 3D reconstructed image obtained from CT scans could accurately reflect the actual glenoid version measured in cadavers. Considering these reports, the authors [13] doubted whether there would be any measurement difference between those taken from axial 2D CT images and those from a 3D reconstructed image. Also, if a difference was found, the 3D reconstructed image would likely be a preferable image type in the clinical field.

Considering that ideal glenoid version can be achieved when the superoinferior axis of glenoid-that is, the coronal plane of the scapula - is orthogonal to the axis of a 2D CT scanner, this study suggested that it is not easy to match this axis in real-world clinical circumstances. Patients may experience difficulties maintaining the necessary position due to pain, anxiety, medical comorbidities, or body habitus. Technicians might face challenges in positioning patients in the right position due to variations among patients' spine curvatures or the anterior tilt of the glenoid itself. As a result, the scanning process may proceed without proper perception of the patient position, which would make it difficult to obtain an accurate scapula axis and which can lead to inaccurate measurements of the glenoid version. Indeed, a difference of $38.4^{\circ}$, which is quite a large variation from $90^{\circ}$, was observed in this study.

The glenoid version is mostly measured on axial 2D CT scans. To assess the difference of the glenoid version measured at different levels, the glenoid version was measured not only at the midpoint of the glenoid, but at levels superior and inferior to the center of the glenoid. From this, it was revealed that the glenoid version was different depending on the level at which measurements are performed. Lewis and Armstrong [25] reported different glenoid version measurements in a different series of glenoid heights collected from 3D CT scans. The tilting angle of the glenoid was also reported to be different in five planes in a 3D image study that adopted MRI by Inui et al. [15]. Furthermore, the glenoid surface itself possesses the shape of a superior-to-inferior spiraling twist $[15,26]$. In this study, a statistically significant difference was found between 2D CT scan images and a 3D reconstructed image at all three levels assessed. This suggests that, by measuring with a $3 \mathrm{D}$ reconstructed image, more accurate measurements can be obtained. Also, this study showed an approximately $4^{\circ}$ difference between measurements at the upper one-third and center of the lower circle of the glenoid. A report by Inui et al. [15] showed the tendency for an increase in retroversion when measurements were conducted superiorly, although the measurement level was different from that in this study.

This study had some limitations. The sample size of each group was relatively small. Because glenoids with deformities were excluded from this study, more studies should be completed to validate our measurement method in patients with arthritis or other defects of the glenoid. However, by measuring the glenoid version on the 3D reconstructed image, no effect of patient positioning was apparent, which is a strength of this research.

In this study, by accurately selecting the transverse planes on the $3 \mathrm{D}$ reconstructed image, comparison with axial 2D CT images was possible. This study has significant value to facilitate clini- 
cal improvement given the availability of easy access to the $3 \mathrm{D}$ reconstruction program. Also, it is meaningful that the angular difference between the scapular body axis and 2D CT slice axis was analyzed. In this investigation, the glenoid version was measured differently between axial 2D CT scans and a 3D reconstructed image at three levels. The $3 \mathrm{D}$ reconstructed image provided more accurate glenoid version data relative to the $2 \mathrm{D} C \mathrm{CT}$ scans. Finally, the glenoid version is measured differently at different levels.

\section{ORCID}

Hyungsuk Kim

Chang Hyun Yoo

Soo Bin Park

Hyun Seok Song https://orcid.org/0000-0003-3400-0263

https://orcid.org/0000-0002-6512-0914

https://orcid.org/0000-0002-6555-5803

https://orcid.org/0000-0002-7844-2293

\section{REFERENCES}

1. Yian EH, Werner CM, Nyffeler RW, et al. Radiographic and computed tomography analysis of cemented pegged polyethylene glenoid components in total shoulder replacement. J Bone Joint Surg Am 2005;87:1928-36.

2. Tétreault P, Krueger A, Zurakowski D, Gerber C. Glenoid version and rotator cuff tears. J Orthop Res 2004;22:202-7.

3. Crockett HC, Gross LB, Wilk KE, et al. Osseous adaptation and range of motion at the glenohumeral joint in professional baseball pitchers. Am J Sports Med 2002;30:20-6.

4. Weishaupt D, Zanetti M, Nyffeler RW, Gerber C, Hodler J. Posterior glenoid rim deficiency in recurrent (atraumatic) posterior shoulder instability. Skeletal Radiol 2000;29:204-10.

5. Wirth MA, Seltzer DG, Rockwood CA Jr. Recurrent posterior glenohumeral dislocation associated with increased retroversion of the glenoid: a case report. Clin Orthop Relat Res 1994;98-101.

6. Friedman RJ, Hawthorne KB, Genez BM. The use of computerized tomography in the measurement of glenoid version. J Bone Joint Surg Am 1992;74:1032-7.

7. Churchill RS, Brems JJ, Kotschi H. Glenoid size, inclination, and version: an anatomic study. J Shoulder Elbow Surg 2001;10:327-32.

8. Shapiro TA, McGarry MH, Gupta R, Lee YS, Lee TQ. Biomechanical effects of glenoid retroversion in total shoulder arthroplasty. J Shoulder Elbow Surg 2007;16 Suppl 3:S90-5.

9. van de Bunt F, Pearl ML, Lee EK, Peng L, Didomenico P. Glenoid version by CT scan: an analysis of clinical measurement error and introduction of a protocol to reduce variability. Skele- tal Radiol 2015;44:1627-35.

10. Nyffeler RW, Sheikh R, Atkinson TS, Jacob HA, Favre P, Gerber C. Effects of glenoid component version on humeral head displacement and joint reaction forces: an experimental study. J Shoulder Elbow Surg 2006;15:625-9.

11. Wong AS, Gallo L, Kuhn JE, Carpenter JE, Hughes RE. The effect of glenoid inclination on superior humeral head migration. J Shoulder Elbow Surg 2003;12:360-4.

12. Das SP, Ray GS, Saha AK. Observations on the tilt of the glenoid cavity of scapula. J Anat Soc India 1966;15:114-8.

13. Kwon YW, Powell KA, Yum JK, Brems JJ, Iannotti JP. Use of three-dimensional computed tomography for the analysis of the glenoid anatomy. J Shoulder Elbow Surg 2005;14:85-90.

14. Nyffeler RW, Jost B, Pfirrmann CW, Gerber C. Measurement of glenoid version: conventional radiographs versus computed tomography scans. J Shoulder Elbow Surg 2003;12:493-6.

15. Inui H, Sugamoto K, Miyamoto T, Machida A, Hashimoto J, Nobuhara K. Evaluation of three-dimensional glenoid structure using MRI. J Anat 2001;199 Pt 3:323-8.

16. Lowe JT, Testa EJ, Li X, Miller S, DeAngelis JP, Jawa A. Magnetic resonance imaging is comparable to computed tomography for determination of glenoid version but does not accurately distinguish between Walch B2 and C classifications. J Shoulder Elbow Surg 2017;26:669-73.

17. Bryce CD, Davison AC, Lewis GS, Wang L, Flemming DJ, Armstrong AD. Two-dimensional glenoid version measurements vary with coronal and sagittal scapular rotation. J Bone Joint Surg Am 2010;92:692-9.

18. Budge MD, Lewis GS, Schaefer E, Coquia S, Flemming DJ, Armstrong AD. Comparison of standard two-dimensional and three-dimensional corrected glenoid version measurements. J Shoulder Elbow Surg 2011;20:577-83.

19. Yushkevich PA, Piven J, Hazlett HC, et al. User-guided 3D active contour segmentation of anatomical structures: significantly improved efficiency and reliability. Neuroimage 2006;31:111628.

20. Rouleau DM, Kidder JF, Pons-Villanueva J, Dynamidis S, Defranco M, Walch G. Glenoid version: how to measure it? Validity of different methods in two-dimensional computed tomography scans. J Shoulder Elbow Surg 2010;19:1230-7.

21. Schrumpf M, Maak T, Hammoud S, Craig EV. The glenoid in total shoulder arthroplasty. Curr Rev Musculoskelet Med 2011;4:191-9.

22. Williams GR Jr, Wong KL, Pepe MD, et al. The effect of articular malposition after total shoulder arthroplasty on glenohumeral translations, range of motion, and subacromial impingement. J Shoulder Elbow Surg 2001;10:399-409. 
23. Farron A, Terrier A, Büchler P. Risks of loosening of a prosthetic glenoid implanted in retroversion. J Shoulder Elbow Surg 2006;15:521-6.

24. Bokor DJ, O’Sullivan MD, Hazan GJ. Variability of measurement of glenoid version on computed tomography scan. J Shoulder Elbow Surg 1999;8:595-8.
25. Lewis GS, Armstrong AD. Glenoid spherical orientation and version. J Shoulder Elbow Surg 2011;20:3-11.

26. Monk AP, Berry E, Limb D, Soames RW. Laser morphometric analysis of the glenoid fossa of the scapula. Clin Anat 2001; 14:320-3. 